\title{
MiR-137 inhibits cervical cancer progression via down-modulating Notch1 and inhibiting the PI3K/AKT/mTOR signaling pathway
}

\author{
Ying Gui ${ }^{1}$, Lina Wang ${ }^{2}$, Zhihong Huang ${ }^{1}$ \\ ${ }^{1}$ Nursing \& Health School of Henan University, Kaifeng, China; ${ }^{2}$ Department of Gynecology, Huaihe Hospital of Henan University, Kaifeng, China \\ Contributions: (I) Conception and design: Z Huang; (II) Administrative support: Z Huang; (III) Provision of study materials or patients: Y Gui; (IV) \\ Collection and assembly of data: L Wang; (V) Data analysis and interpretation: Y Gui; (VI) Manuscript writing: All authors; (VII) Final approval of \\ manuscript: All authors. \\ Correspondence to: Zhihong Huang. Nursing and Health School of Henan University, Jinming Street, Kaifeng 475004, China. Email: hzhh66@163.com.
}

\begin{abstract}
Background: Cervical cancer (CC) is frequently diagnosed, and accounts for a large proportion of cancerrelated mortality worldwide. Therefore, it is an urgent need to explore the mechanisms of CC progression and seek new therapeutic targets. We know that microRNA-137 (miR-137) is involved in CC progression; however, it's mechanism in CC has not yet been clarified.

Methods: Quantitative real-time PCR (qRT-PCR) was applied to detect the expressions of miR-137 and Notch homolog 1 messenger RNA (Notch1 mRNA) in CC cells. Cell Counting Kit-8 (CCK-8) and Transwell assay were utilized to monitor the proliferation, invasion, and migration of CC cells. The binding relationship of miR-137 with Notch1 was detected by dual luciferase reporter gene assay. Western blot was used to determine the expressions of Notch1, p-AKT/AKT, and p-mTOR/mTOR signaling pathways.

Results: The results showed that miR-137 was down-regulated and Notch1 was up-regulated in CC cells. MiR-137 was reversely correlated with Notch1, and Notch1 partially reversed the promotion of miR-137 silencing on malignant phenotype of CC cells. Furthermore, in vitro experiments confirmed that miR-137 partially impeded CC progression via regulation of the Notch1 and PI3K/AKT/mTOR axis.

Conclusions: MiR-137 targets Notch1 and regulates CC through the PI3K/Akt/mTOR pathway. The miR-137/Notch1 axis may be a promising target for CC patients, and these findings can provide a reference for the clinical treatment of CC.
\end{abstract}

Keywords: MicroRNA-137 (miR-137); Notch1; cervical cancer (CC); proliferation; metastasis

Submitted Apr 20, 2021. Accepted for publication Aug 12, 2021.

doi: $10.21037 /$ tcr-21-1049

View this article at: https://dx.doi.org/10.21037/tcr-21-1049

\section{Introduction}

Cervical cancer (CC) is a common clinical diagnosis of female reproductive system cancer, which is second only to breast cancer (1). It is a serious threat to the health and life of affected women (2). In the past few decades, the strengthening of histopathological examination and the improvement of early diagnosis of cervical cancer have significantly reduced the morbidity and mortality of the disease (3). In recent years, the molecular mechanism related to cervical cancer has been widely concerned, but the development process of cervical cancer is not completely clear.

MicroRNAs (miRNAs) are non-coding RNAs engaged in the progression of multiple cancers (4). The miRNAs exert their influence by modulating gene expression. When miRNAs bind to the 3' UTR of messenger RNAs (mRNAs), they can induce the degradation or inhibit the translation of mRNAs $(5,6)$. The miRNAs serve as diagnostic indicators and prognostic biomarkers for various cancers (7). Mounting evidence has shown that miR-137 may be a new prognostic marker of CC. Abundance of miR-137 is strongly related 
to the proliferation, invasion, and migration $\mathrm{CC}$ cells, and miR-137 may become a new prognostic indicator for CC therapy (8). Miao et al. showed that down-regulated miR137 in CC cells accelerated apoptosis and impeded cell proliferation and metastasis via targeting GREM1 (9). In this study, we found a new signaling pathway through which miR-137 blocked the progression of CC.

The miRNAs conduct their role via targeting and regulating the expression of mRNAs $(5,6)$. Notch and its related molecules are important members of the signal receptor protein family, which were firstly discovered in vertebrates and invertebrates. Notch signals can realize communication, signal transduction, and transcription through local cell-to-cell interactions, and control cell proliferation, apoptosis, and other activities. The signal pathway was mainly composed of receptors, ligands, CBF$1 / \mathrm{RBP}-\mathrm{j}_{\mathrm{K}}, \mathrm{Su}(\mathrm{H}), \mathrm{Lag}-1$ (CSL) proteins, and signal effector molecules. In the human body, the Notch pathway has 4 receptors, which are involved in tumor activity through their interaction with ligands. Among them, the Notch1 receptor has been most extensively studied to date. Some researchers have conducted immunohistochemical (IHC) analyses, confirming that highly-expressed Notch 1 in various tumor cells was related to some pathological features and prognosis (10-12). Sun et al. asserted that Notch1 can enhance the proliferation of CC cells, which indicated that Notch1 may serve as a promoter in CC progression (13). Wei et al. showed that miR-34c-5p can impede the proliferation while promoting apoptosis of CC cells via targeting Notch1 (14). Previous studies have confirmed that miR-137 can impede CC progression and pancreatic cancer by targeting Notch1 $(15,16)$. In this study, we firstly detected the direct interaction of miR-137 with Notch1 in CC.

The PI3K/AKT/mTOR signaling pathway is strongly associated with cell differentiation and proliferation (17-20). Recent studies have indicated that blocking of the PI3K/ $\mathrm{AKT} / \mathrm{mTOR}$ is effective to treat CC (21). In the present study, the activity of the PI3K/AKT/mTOR pathway was investigated to confirm whether miR-137/Notch1 wield an influence via this pathway. In the present study, we investigated the relationship of miR-137 with Notch1 in CC progression, and determined whether miR-137 modulated the metastasis and proliferation of CC cells via Notch1/PI3K/AKT/mTOR.

We present the following article in accordance with the MDAR reporting checklist (available at https://dx.doi. org/10.21037/tcr-21-1049).

\section{Methods}

\section{CC tissues}

A total of 40 pairs of fresh CC tissues and paired adjacent tissues were all obtained from cancer tissue specimens of the Huaihe Hospital of Henan University. Before sample collection, no participants received chemotherapy, immunotherapy, hormone therapy, or radiotherapy. Eligible patients provided written informed consent (22). All procedures performed in this study involving human participants were in accordance with the Declaration of Helsinki (as revised in 2013). The study was approved by institutional ethic board of Huaihe Hospital of Henan University. The collected tissues were stored at $-80{ }^{\circ} \mathrm{C}$ for subsequent analyses.

\section{Cell culture and transfection}

We procured two CC cell lines (SiHa and HeLa) and human cervical immortalized squamous cells (ECT1/E6E7) from American Type Culture Collection (ATCC, Rockville, USA). The cells were cultured in Dulbecco's modified eagle medium (DMEM, Hyclone, Logan, UT, USA) containing $10 \%$ fetal bovine serum (FBS, Sigma-Aldrich, St. Louis, MO, USA) and antibiotics. The cells were stored in a humidified incubator with $5 \% \mathrm{CO}_{2}$ at $37^{\circ} \mathrm{C}$.

Inhibitors and mimics of miR-137 were procured from Shanghai Gene Pharmaceutical Co., Ltd. (Shanghai, China) for down-regulation and overexpression of miR-137. The negative control mimics NC/inhibitor NC was provided by Guangzhou Nuclear Biology Co., Ltd (Guangzhou, China). The miR-137 inhibitor, mimics, and corresponding negative control were transfected into SiHa and HeLa with liposome (Invitrogen, Carlsbad, CA, USA) for $48 \mathrm{~h}$.

\section{Quantitative real-time polymerase chain reaction}

The DNA samples were incubated with deoxyribonuclease at $37^{\circ} \mathrm{C}$ for $90 \mathrm{~min}$ to remove genomic DNA. In order to detect the expressions of miR-137 and Notch1 mRNA, the total RNA was reversely transcribed into circular DNA (cDNA), and quantitative real-time polymerase chain reaction (qRT-PCR) was done with glyceraldehyde 3 -phosphate dehydrogenase (GAPDH) as the internal reference. The expression of miR-137 was detected by Allin-One ${ }^{\mathrm{TM}}$ miRNA qRT-PCR detection kit (GeneCopoeia, Inc., Rockville, MD, USA). We added U6 as an endogenous 
reference of miR-137. The $\mathrm{Ct}$ values were normalized by the $2^{-\Delta \Delta C T}$ method.

\section{Cell Counting Kit-8 (CCK-8) assay}

CCK-8 (Sigma) was utilized to monitor cell proliferation and its proliferation ability. The CC cells (5,000/well) were seeded in 96 well plate overnight. At $24 \mathrm{~h}$ after transfection, $15 \mathrm{~mL}$ of CCK-8 solution was incubated with living CC cells at room temperature for $2 \mathrm{~h}$. The absorbance was analyzed at $450 \mathrm{~nm}$ using a flat microplate reader (Molecular Devices, San Jose, CA, USA). And all experiments were performed three times in different groups.

\section{Transwell migration and invasion assay}

The migration and invasion ability of CC cells were analyzed by using Transwell chambers with an $8-\mathrm{mm}$ filter (Corning Co., Corning, NY, USA). In the Transwell migration assay, CC cells $\left(1 \times 10^{4} / 100 \mathrm{~mL}\right.$ serum-free medium) were inoculated into the uncoated upper cavity. At $24 \mathrm{~h}$ after transfection, FBS (10\%) was added to the medium to fill the lower cavity as a chemotactic inducer. After $24 \mathrm{~h}$ of incubation, unmigrated CC cells on the upper surface were scraped with cotton swabs, fixed with $4 \%$ paraformaldehyde (Sangon Biotech, Shanghai, China), and stained with crystal violet (Sangon Biotech). The number of migrated CC cells in 5 random wells was counted. In the invasion assay, $40 \mathrm{~mL}$ BD Matrigel matrix (BD Biosciences, Becton, Dickinson, and Co., San Jose, CA, USA) was diluted in 1:8 cold water and pre-coated serum-free medium to simulate extracellular matrix, and CC cells were inoculated $\left(7 \times 10^{4} / 100 \mathrm{~mL}\right.$ serum-free medium, $24 \mathrm{~h}$ after transfection). The other steps were similar to those of the migration assay. And all experiments were performed three times in different groups.

\section{Luciferase reporter gene detection}

First, the Notch1 3'-UTR-pGL3-reporter vector (Promega, Madison, WI, USA) was constructed. Then, the vector and miR-137 were co-transfected into HEK293T. At 48 h after transfection, the luciferase activity was detected by the dual luciferase reporter system (Promega).

\section{Western blot}

Total proteins were extracted with radioimmunoprecipitation assay (RIPA) lysis buffer (Beyotime, Shanghai, China). The protein concentration was measured using protein extraction kit (Thermo Fisher Scientific Inc., Waltham, MA, USA). Then, the protein was isolated by sodium dodecyl sulfate polyacrylamide gel electrophoresis (SDS-PAGE) and transferred to the NC membrane. Following blocking with $5 \%$ skim milk powder at $37^{\circ} \mathrm{C}$ for $1 \mathrm{~h}$, the primary antibody was added for incubation at $4{ }^{\circ} \mathrm{C}$ overnight, then the secondary antibody at $37^{\circ} \mathrm{C}$ for $1 \mathrm{~h}$. Ultimately, enhanced chemiluminescence (ECL) kit (Pierce; Thermo Fisher Scientific, Inc.) was used for protein detection, which was then quantified by Image J software (National Institutions of Health, Bethesda, MD, USA). And all experiments were performed three times in different groups.

\section{Statistical analyses}

Statistical data were expressed as means \pm standard deviation (SD). Student's $t$-test and one-way analysis of variance (ANOVA) were also used for statistical analysis.

\section{Results}

MiR-137 was down-modulated in CC cells, while Notch1 expression was up-regulated

We performed qRT-PCR to determine expressions of miR-137 and Notch 1 in CC cells. Consistent with the findings of previous researches $(9,13)$, miR-137 level in CC cells was notably lower and Notch1 was higher than that in adjacent tissues and human cervical epithelial cells (Figure 1A-1D).

\section{MiR-137 deletion accelerated metastasis and proliferation of CC cells}

The function loss experiment revealed that the enrichment of miR-137 in SiHa and HeLa cells transfected with miR137 inhibitor was significantly lower than in the blank and inhibitor NC groups (Figure 2A,2B). The proliferation of SiHa and HeLa cells transfected with miR-137 inhibitor increased more than in the control group (Figure $2 C, 2 D$ ). As displayed in Figure $2 E$ and $2 F$, the migration and invasion assay showed that impeded miR-137 accelerated cell metastasis. In summary, miR-137 can impede the metastasis and proliferation of CC cells. Therefore, miR-137 had an anti-cancer effect in CC cells, which was consistent with previous reports $(9)$. 


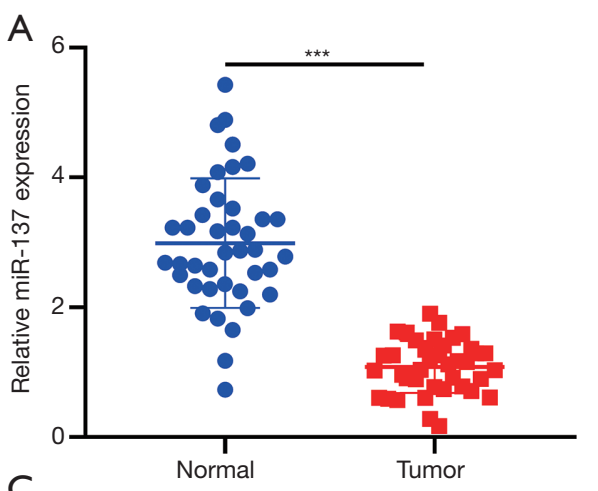

C

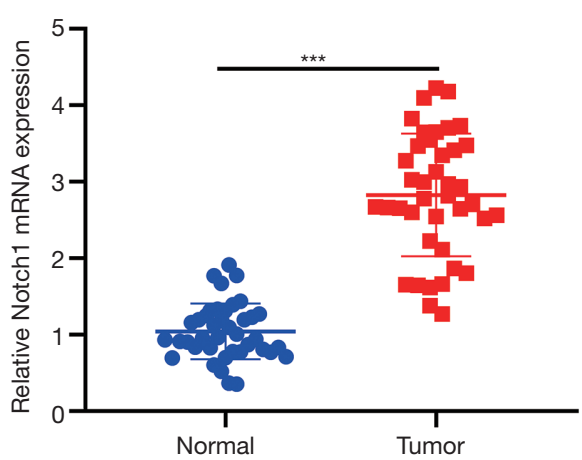

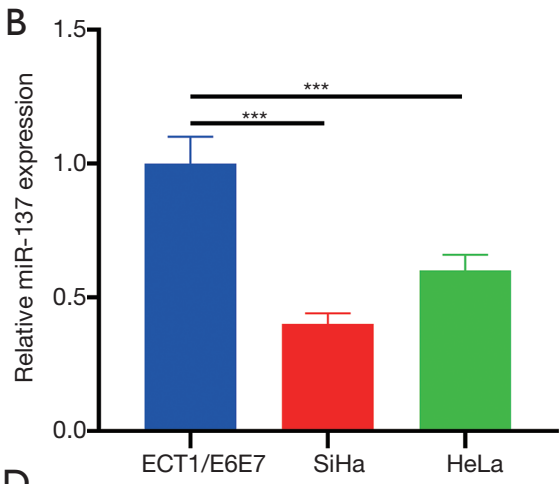

D

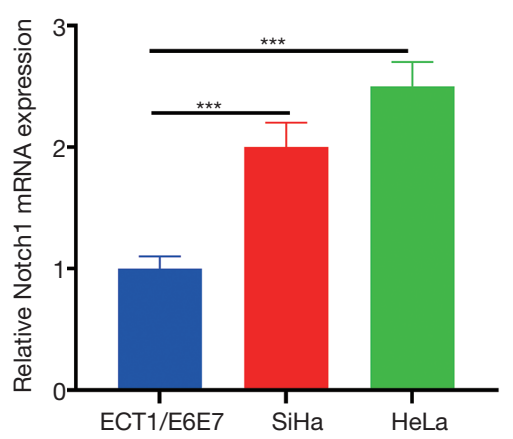

Figure 1 MiR-137 was down-regulated in CC tissue and cells. (A,B) The expressions of miR-137 in 40 CC tissues and corresponding normal tissues were detected by qRT-PCR; (C,D) the expressions of Notch1 in 40 CC tissues and corresponding normal tissues were detected by qRT-PCR. ***, $\mathrm{P}<0.001$. miR-137, microRNA-137; CC, cervical cancer; qRT-PCR, real-time quantitative polymerase chain reaction.

\section{Notch1 was the target of miR-137 in CC and was reversely modulated by miR-137}

As shown in Figure 3A, the 3 'UTR of Notch1 mRNA bound to the "seed" site of miR-137. In HEK293T cells cotransfected with miR-137 mimics and Notch1-WT plasmid, luciferase activity was significantly decreased, while in cells co-transfected with miR-137 mimics and Notch1-MUT, the luciferase activity exhibited notable change, indicating that miR-137 binds directly to Notch1 (Figure 3B). To explore the regulation mechanism of miR-137 and Notch1 in SiHa and HeLa cells, qRT-PCR was used to detect Notch1 after miR-137 mimics or miR-137 inhibitor was transfected into $\mathrm{SiHa}$ and HeLa cells. Overexpressed miR-137 was shown to significantly downregulate Notch 1 expression. Conversely, the deletion of miR-137 upregulated Notch1 levels in $\mathrm{SiHa}$ and HeLa cells (Figure 3C,3D). In addition, Pearson's correlation analysis showed that the abundance of Notch1 was inversely related with miR-137 in $40 \mathrm{CC}$ tissues (Figure 3E). Therefore, Notch1 was reversely correlated with miR-137.

\section{Notch1 attenuated the promotion of miR-137 deletion on metastasis and proliferation of CC cells}

Addition of miR-137 inhibitors upregulated Notch1, while co-transfection of si-Notch1 and miR-137 inhibitors decreased Notch1 expression (Figure 4A). The influence of miR-137 deletion on cell proliferation was partially counteracted by Notch1 (Figure 4B,4C). The Transwell assay showed that the intervention of Notch 1 partially canceled the promotion of miR-137 inhibitors on $\mathrm{SiHa}$ and HeLa cell metastasis (Figure 4D,4E). It was also shown that miR-137 impeded the metastasis of CC cells at least partially by targeting Notch 1 . In general, miR-137 inhibited the progression of CC at least by targeting Notch1. 

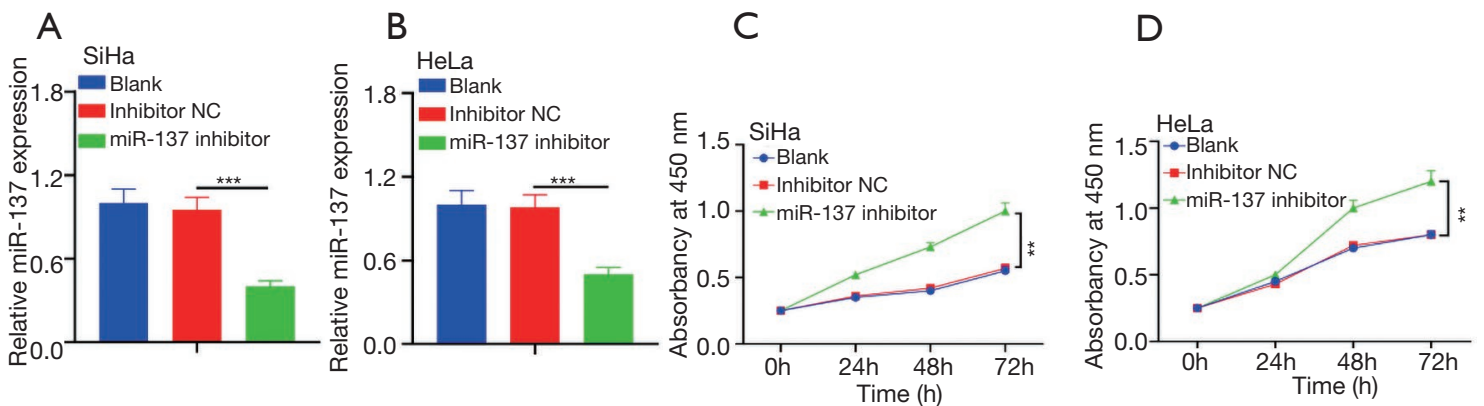

$E$

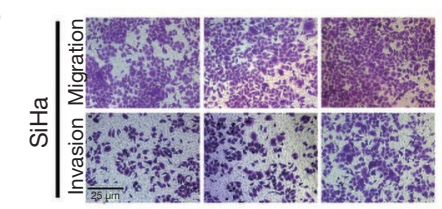

$\mathrm{F}$
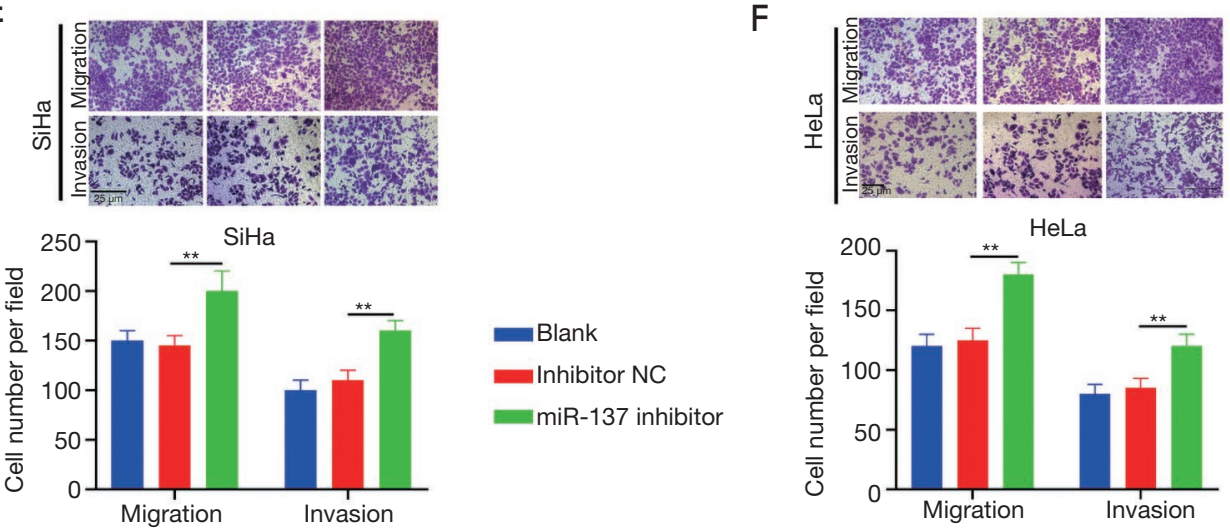

Figure 2 MiR-137 impeded proliferation and metastasis of CC cells. (A,B) After 24 h transfection of SiHa and HeLa cells, qRT-PCR was used to detect the knockout efficiency of miR-137 inhibitors on SiHa and HeLa cells; (C,D) cell proliferation was detected by CCK8 method. CC cells were inoculated into 96-well plates, the absorbance was measured at $0,24,48$, and $72 \mathrm{~h}$ after transfection, and the cell proliferation curve was drawn; (E,F) at $24 \mathrm{~h}$ after transfection, the migration and invasion ability of CC cells were detected by Transwell migration and invasion assay. Crystal violet staining, the magnification was 100x. ${ }^{* *}, \mathrm{P}<0.01,{ }^{* * *}, \mathrm{P}<0.001$. miR-137, microRNA-137; CC, cervical cancer; qRT-PCR, real-time quantitative polymerase chain reaction; CCK-8, Cell Counting Kit-8.

\section{Notch1 gene knockout eliminated the promotion of miR- 137 deletion on the PI3K/AKT/mTOR patbway}

Deletion of miR-137 upregulated the phosphorylation levels of AKT and mTOR, while the addition of si-Notch1 blocked AKT and mTOR at phosphorylation level (Figure 5A,5B). MiR-137 impeded the proliferation of CC cells via upmodulating Notch1 and blocking PI3K/AKT/mTOR.

\section{Discussion}

In early stage of onset, CC demonstrates no notable symptoms. It is often misdiagnosed, resulting in a low early diagnosis rate. Although comprehensive treatment including surgical resection, radiotherapy, and chemotherapy has made great progress, the therapeutic effect on advanced patients has remained unsatisfactory. Therefore, it is imperative to study and identify key regulatory factors for early diagnosis, therapeutic effect, and prognosis. In this study, miR-137 was show to bind to Notch1, and block the PI3K/AKT/ mTOR signaling pathway via down-regulating Notch1, thus blocking the proliferation and metastasis of CC cells.

Increasing articles have pointed out that disordered miRNAs were related to the tumorigenesis and progression of cancer $(23,24)$. The expression of miR-137 has been shown to be decreased in multiple tumors. Ding et al. showed that lowly-expressed miR-137 impeded the invasion and proliferation of prostate cancer cells via up-modulating MRGBP (25). Bi et al. claimed that the abundance of miR137 in plasma and exosome of colorectal cancer patients was decreased, and miR-137 may impede the proliferation and metastasis of CC cells via increasing the abundance of TCF4 (26). Chen asserted that miR-137 can impede the growth of CC cells (8). The qRT-PCR revealed that miR137 exhibited a higher enrichment in CC cells than adjacent tissues. The down-modulation of miR-137 expression in CC 

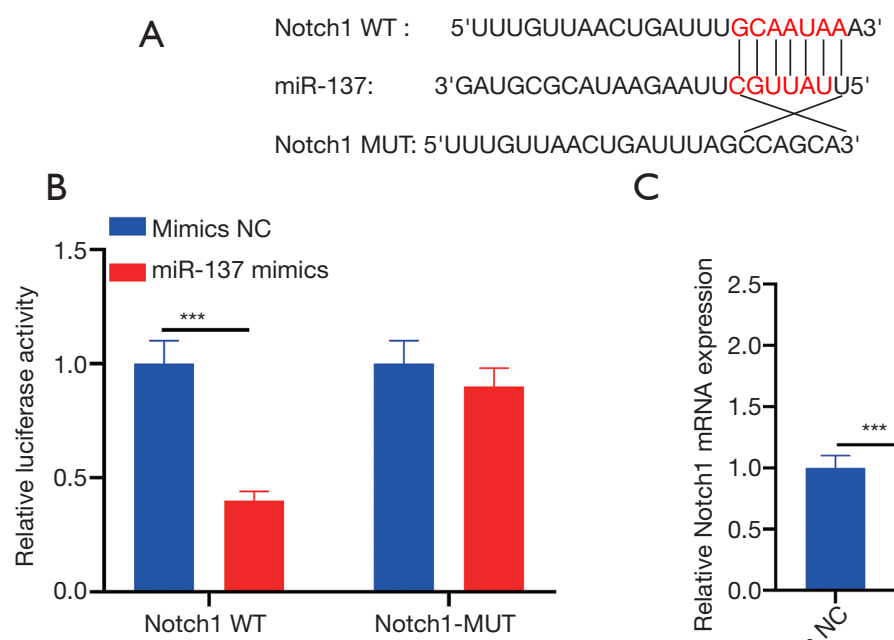

C
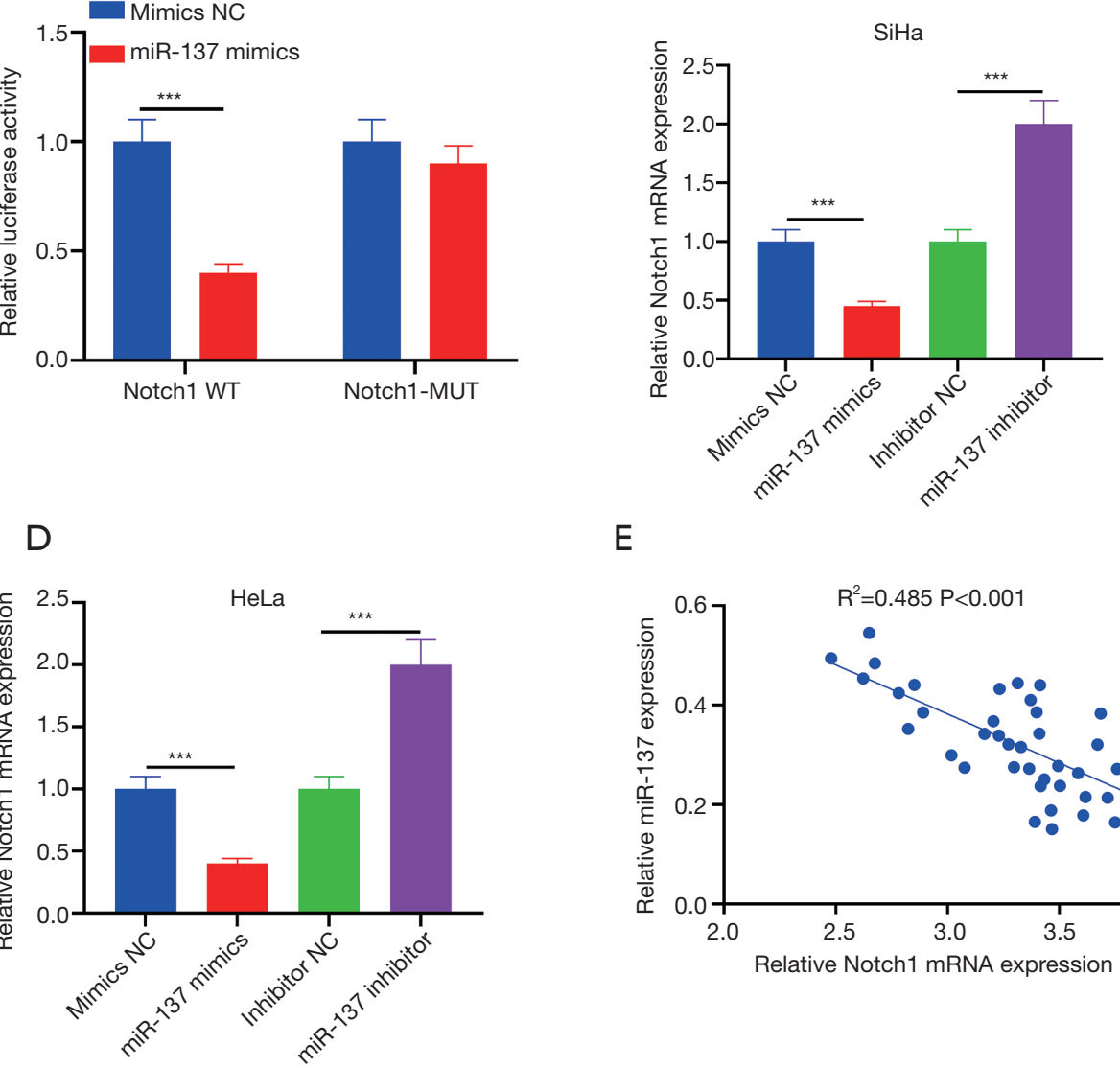

$E$

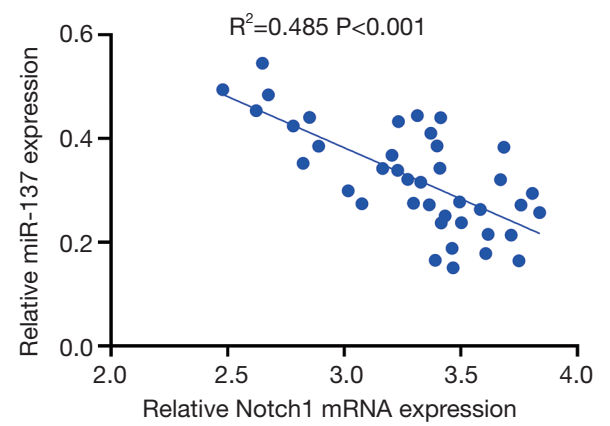

Figure 3 Notch1 was the target of miR-137 (A) TargetScan online software was used to predict the binding site between miR-137 and Notch1; (B) SiHa and HeLa cells were co-transfected with WT and MUT and NC mimics or miR-137 mimics for $48 \mathrm{~h}$ to detect luciferase activity; (C,D) Notch1 expression was detected in CC cells transfected with miR-137 mimics and inhibitors; (E) The relationship between miR-137 and Notch1 expression was analyzed in CC tissues. ***, $\mathrm{P}<0.001$. Notch1, Notch homolog 1; miR-137, microRNA-137; CC, cervical cancer; WT, wild type; MUT, mutant; NC, negative control

was in accord with previous studies. Miao et al. found that GREM1 was the target gene of miR-137, which impeded the progression of CC via GREM1 (9). In this study, another target of miR-137, Notch1, was predicted by TargetScan (http://www.targetscan.org/vert_71/), and the relationship of miR-137 with Notch1 was also verified. In SiHa and HeLa cells, miR-137 negatively regulated Notch 1 .

Unregulated NOTCH signals with mutation or abnormal expression of the NOTCH receptor gene have been reported in solid tumors, including CC $(27,28)$. Evidence has shown that NOTCH1 is related to the proliferation and invasion of CC. Hu et al. revealed that NOTCH1 modulated the biological process of CC cells (29). Song et al. claimed that NOTCH1 was overexpressed in CC, suggesting its invasive biological behavior (30). Maliekal et al. found that in CC, the two carcinogenic mechanisms by which Notch signaling plays a role in this respect are activation of the PI3K/Akt pathway and the upregulation of c-Myc (31). These studies supported the direct role of NOCTH signaling in CC. Notch1 was up-modulated in CC cells, which was consistent with the findings of previous studies (13). Functional experiments confirmed that Notch1 

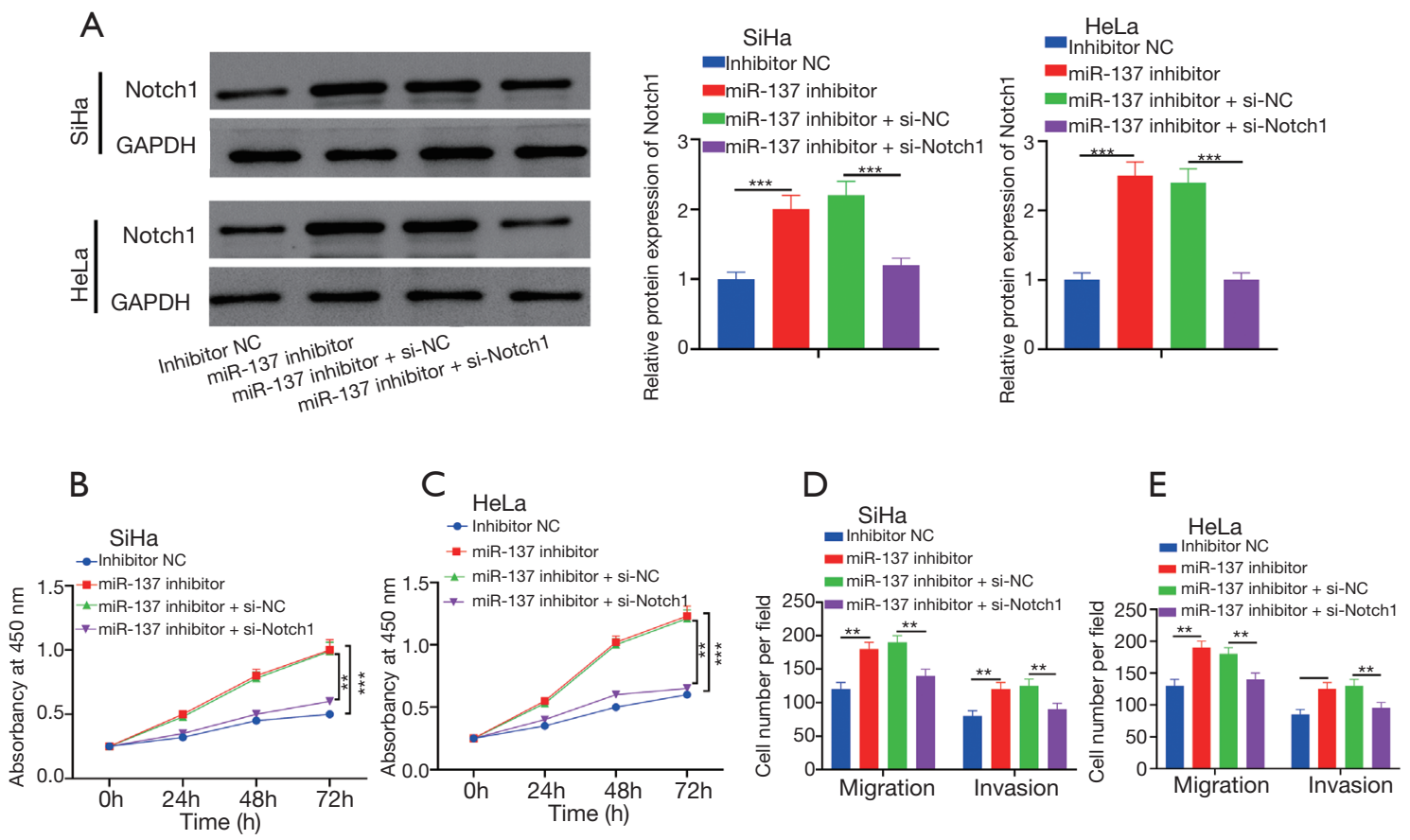

Figure 4 MiR-137 can inhibit the proliferation and metastasis of CC cells by regulating Notch1. SiHa and HeLa cells were transfected with NC inhibitor, miR-137 inhibitor, or miR-137 inhibitor plus si-NC or si-Notch1. (A) Western blot was used to detect the expressions of Notch1 in CC cells $24 \mathrm{~h}$ after transfection; (B,C) CCK-8 assay was used to detect the proliferation of CC cells; (D,E) at $24 \mathrm{~h}$ after transfection, the metastasis of $\mathrm{CC}$ cells was detected by Transwell migration and invasion assay $(\mathrm{n}=3)$. ${ }^{* *}, \mathrm{P}<0.01$; *** $\mathrm{P}<0.001$. Notch1, Notch homolog 1; miR-137, microRNA-137; NC, negative control; CC, cervical cancer; CCK-8, Cell Counting Kit-8.
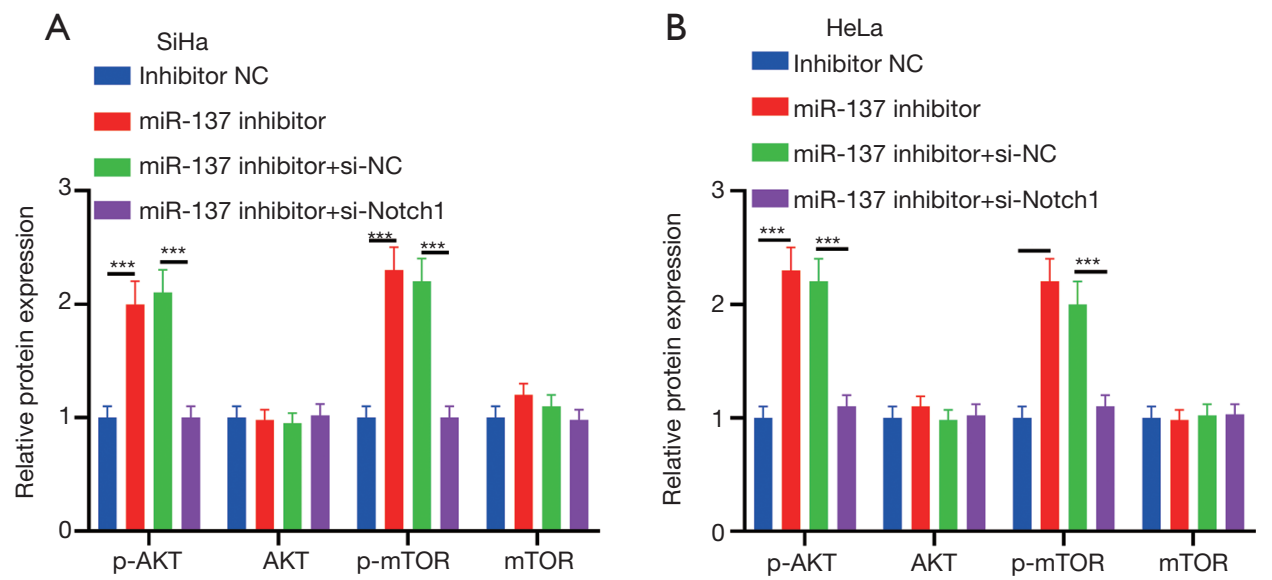

Figure 5 MiR-137 impeded progression of the Notch1/PI3K/AKT/mTOR pathway to inhibit CC progression (A,B) Western blot was used to detect AKT, AKT phosphorylation, mTOR, and mTOR phosphorylation in SiHa and HeLa cells of transfection NC inhibitor, miR-137 inhibitor, or miR-137 inhibitor + si-NC or si-Notch1 for 24 h. ${ }^{* *}, \mathrm{P}<0.001$. miR-137, microRNA-137; Notch1, Notch homolog 1; PI3K, phosphatidylinositol 3-kinase;mTOR, mammalian rapamycin target protein; NC, negative control. 
could partially reverse the impact of miR-137 on CC. Thus, miR-137 negatively modulates Notch1 to inhibit the metastasis and proliferation of CC cells.

As one of the most characteristic kinase-grade pathways, PI3K/AKT wields a central role in cancer progression $(32,33)$. In CC cells, PI3K/AKT exerts an influence in biological processes $(34,35)$. The PI3K/Akt pathway was shown to have a close relationship with CC progression (36), and was shown to be another a potential target for CC $(37,38)$. The low expression of miR-137 increased the expression of p-PI3K/PI3K and p-AKT/AKT, while the low expression of Notch1 resulted in the opposite effect. Collectively, we speculate that miR-137 can impede PI3K/ AKT via Notch1 in CC cells.

\section{Conclusions}

This study clarified the antitumor effect of miR-137 in CC. Down-regulation of miR-137 activates PI3K/AKT/mTOR via up-modulation of Notch1, and impedes metastasis and proliferation of CC cells. Therefore, miR-137/Notch1 may be a potential therapeutic target for CC. Unfortunately, this study still had some shortcomings, and tumor formation experiments are required to further verify the role of miR137/Notch1 in CC. Other downstream targets of miR-137 in CC need to be further explored.

\section{Acknowledgments}

Funding: None.

\section{Footnote}

Reporting Checklist: The authors have completed the MDAR reporting checklist. Available at https://dx.doi. org/10.21037/tcr-21-1049

Data Sharing Statement: Available at https://dx.doi. org/10.21037/tcr-21-1049

Conflicts of Interest: All authors have completed the ICMJE uniform disclosure form (available at https://dx.doi. org/10.21037/tcr-21-1049). The authors have no conflicts of interest to declare.

Ethical Statement: The authors are accountable for all aspects of the work in ensuring that questions related to the accuracy or integrity of any part of the work are appropriately investigated and resolved. All procedures performed in this study involving human participants were in accordance with the Declaration of Helsinki (as revised in 2013). The study was approved by institutional ethic board of Huaihe Hospital of Henan University. Eligible patients provided written informed consent.

Open Access Statement: This is an Open Access article distributed in accordance with the Creative Commons Attribution-NonCommercial-NoDerivs 4.0 International License (CC BY-NC-ND 4.0), which permits the noncommercial replication and distribution of the article with the strict proviso that no changes or edits are made and the original work is properly cited (including links to both the formal publication through the relevant DOI and the license). See: https://creativecommons.org/licenses/by-nc-nd/4.0/.

\section{References}

1. Schiffman M, Solomon D. Clinical practice. Cervicalcancer screening with human papillomavirus and cytologic cotesting. N Engl J Med 2013;369:2324-31.

2. Pimple S, Mishra G, Shastri S. Global strategies for cervical cancer prevention. Curr Opin Obstet Gynecol 2016;28:4-10.

3. Bansal N, Herzog TJ, Shaw RE, et al. Primary therapy for early-stage cervical cancer: radical hysterectomy vs radiation. Am J Obstet Gynecol 2009;201:485.e1-9.

4. Croce CM, Calin GA. miRNAs, cancer, and stem cell division. Cell 2005;122:6-7.

5. Ambros V. The functions of animal microRNAs. Nature 2004;431:350-5.

6. Calin GA, Croce CM. MicroRNA signatures in human cancers. Nat Rev Cancer 2006;6: 857-66.

7. Liu R, Chen X, Du Y, et al. Serum microRNA expression profile as a biomarker in the diagnosis and prognosis of pancreatic cancer. Clin Chem 2012;58:610-8.

8. Chen Y, Gen Y, Huang J, et al. CircNEIL3 promotes cervical cancer cell proliferation by adsorbing miR-137 and upregulating KLF12. Cancer Cell Int 2021;21:34.

9. Miao H, Wang N, Shi LX, et al. Overexpression of mircoRNA-137 inhibits cervical cancer cell invasion, migration and epithelial-mesenchymal transition by suppressing the TGF- $\beta$ /smad pathway via binding to GREM1. Cancer Cell Int 2019;19:147.

10. Liang T, Guo Q, Li L, et al. MicroRNA-433 inhibits migration and invasion of ovarian cancer cells via targeting Notch1. Neoplasma 2016;63:696-704.

11. Rui-Huan Gan, Hua Wei, Jing Xie, et al. Notch1 regulates 
tongue cancer cells proliferation, apoptosis and invasion. Cell Cycle 2018;17:216-24.

12. Liu T, Zuo JJ, Li F, et al. LncRNA SNHG1 promotes cell proliferation in laryngeal cancer via Notch1 signaling pathway. Eur Rev Med Pharmacol Sci 2019;23:6562-9.

13. Sun Y, Zhang R, Zhou S, et al. Overexpression of Notch1 is associated with the progression of cervical cancer. Oncol Lett 2015;9:2750-6.

14. Wei H, Wang X, Niu X, et al. miR-34c-5p targets Notch1 and suppresses the metastasis and invasion of cervical cancer. Mol Med Rep 2021;23:120.

15. Xu J, Wu G, Zhao Y, et al. Long Noncoding RNA DSCAM-AS1 Facilitates Colorectal Cancer Cell Proliferation and Migration via miR-137/Notch1 Axis. J Cancer 2020;11:6623-32.

16. Liu PJ, Pan YH, Wang DW, et al. Long non-coding RNA XIST promotes cell proliferation of pancreatic cancer through miR-137 and Notch1 pathway. Eur Rev Med Pharmacol Sci 2020;24:12161-70.

17. Sathe A, Nawroth R. Targeting the PI3K/AKT/ mTOR pathway in bladder cancer. Methods Mol Biol 2018;1655:335-50.

18. LoRusso PM. Inhibition of the PI3K/AKT/mTOR pathway in solid tumors. J Clin Oncol 2016;34:3803-15.

19. Mabuchi S, Kuroda H, Takahashi R, et al. The PI3K/ AKT/mTOR pathway as a therapeutic target in ovarian cancer. Gynecol Oncol 2015;137:173-9.

20. Guerrero-Zotano A, Mayer IA, Arteaga CL. PI3K/AKT/ mTOR: role in breast cancer progression, drug resistance, and treatment. Cancer Metastasis Rev 2016;35:515-24.

21. Li YJ, Wang Y, Wang YY. MicroRNA-99b suppresses human cervical cancer cell activity by inhibiting the PI3K/AKT/mTOR signaling pathway. J Cell Physiol 2019;234:9577-91.

22. Xiaotian Luan, Yankui Wang. LncRNA XLOC_006390 facilitates cervical cancer tumorigenesis and metastasis as a ceRNA against miR-331-3p and miR-338-3p. J Gynecol Oncol. 2018;29:e95.

23. Di Leva G, Garofalo M, Croce CM. MicroRNAs in cancer. Annu Rev Pathol 2014;9:287-314.

24. Zhang B, Pan X, Cobb GP, et al. microRNAs as oncogenes and tumor suppressors. Dev Biol 2007;302:1-12.

25. Ding F, Zhang S, Gao S, et al. MiR-137 functions as a tumor suppressor in pancreatic cancer by targeting MRGBP. J Cell Biochem 2018;119:4799-807.

26. Bi WP, Xia M, Wang XJ. miR-137 suppresses proliferation, migration and invasion of colon cancer cell lines by targeting TCF4. Oncol Lett 2018;15:8744-8.
27. Aster JC, Pear WS, Blacklow SC. The Varied Roles of Notch in Cancer. Annu Rev Pathol 2017;12:245-75.

28. Rodrigues C, Joy LR, Sachithanandan SP, et al. Notch signalling in cervical cancer. Exp Cell Res 2019;385:111682.

29. Lian H, Hui Y, Xiaoping T, et al. Baicalein suppresses the proliferation of human cervical cancer cells via Notch $1 / \mathrm{Hes}$ signaling pathway. J Cancer Res Ther 2019;15:1216-20.

30. Song L, Meng L, Peng J, et al. Clinicopathological significance of Notch1 expression in cervical cancer patients: a meta-analysis. Biomark Med 2016;10:63-72.

31. Maliekal TT, Bajaj J, Giri V,et al. The role of Notch signaling in human cervical cancer: implications for solid tumors. Oncogene 2008;27:5110-4.

32. Manning BD, Cantley LC. AKT/PKB signaling: navigating downstream. Cell 2007;129:1261-74.

33. Stegeman H, Span PN, Kaanders JH. Improving chemoradiation efficacy by PI3-K/AKT inhibition. Cancer Treat Rev 2014;40:1182-91.

34. Wang F, Tan WH, Liu W, et al. Effects of miR-214 on cervical cancer cell proliferation, apoptosis and invasion via modulating PI3K/AKT/mTOR signal pathway. Eur Rev Med Pharmacol Sci 2018;22:1891-8.

35. Tsai JP, Lee CH, Ying TH, et al. Licochalcone A induces autophagy through PI3K/Akt/mTOR inactivation and autophagy suppression enhances licochalcone A-induced apoptosis of human cervical cancer cells. Oncotarget 2015;6:28851-66.

36. Jiang E, Sun X, Kang H, et al. Dehydrocostus lactone inhibits proliferation, antiapoptosis, and invasion of cervical cancer cells through PI3K/Akt signaling pathway. Int J Gynecol Cancer 2015;25:1179-86.

37. Schwarz JK, Payton JE, Rashmi R, et al. Pathway-specific analysis of gene expression data identifies the PI3K/Akt pathway as a novel therapeutic target in cervical cancer. Clin Cancer Res 2012;18:1464-71.

38. Wu J, Chen C, Zhao KN. Phosphatidylinositol 3-kinase signaling as a therapeutic target for cervical cancer. Curr Cancer Drug Targets 2013;13:143-56.

(English Language Editor: J. Jones)

Cite this article as: Gui Y, Wang L, Huang Z. MiR-137 inhibits cervical cancer progression via down-modulating Notch1 and inhibiting the PI3K/AKT/mTOR signaling pathway. Transl Cancer Res 2021;10(8):3748-3756. doi: 10.21037/tcr-21-1049 